\title{
An Experimental Study on Fracture Energy of Plain Concrete
}

\author{
Jaeha Lee ${ }^{1), *}$, and Maria M. Lopez ${ }^{2}$
}

(Received May 22, 2013, Accepted January 6, 2014)

\begin{abstract}
In this study, the concrete fracture energy was obtained using the three point notched beam test method developed by Hillerborg et al. (Cem Concr Res 6(6):773-782, 1976). A total of 12 notched concrete beams were tested under two different loading conditions: constant stroke control and constant crack mouth opening displacement (CMOD) control. Despite individual fracture energies obtained from the two different loading conditions showing some variation, the average fracture energy from both loading conditions was very similar. Furthermore, the results obtained support the idea that a far tail constant " $\mathrm{A}$ " could change the true fracture energy by up to $11 \%$, if it is calculated using CMOD instead of LVDT. The far tail constant " $\mathrm{A}$ ” is determined using a least squares fit onto a straight line according to Elices et al. (Mater Struct 25(148):212-218, 1992) and RILEM report (2007). It was also observed that the selection of the end point can produce variations of the true fracture energy. The end point indicates the point in the experiment at which to stop. An end point of $2 \mathrm{~mm}$ has been recommended, however, in this study other end points were also considered. The final form of the bilinear softening curve was determined based on Elices and Guinea's methods (1992, 1994) and RILEM report (2007). This paper proposes a bilinear stress-crack opening displacement curve according to test results as well as the CEB-FIP model code.
\end{abstract}

Keywords: concrete, fracture energy, bilinear softening curve, tensile behavior.

\section{Introduction}

A few decades ago much research was undertaken to obtain the tensile properties of concrete using various methods such as a cohesive crack (Hillerborg et al. 1976), crack band (Bažant 1976) or the damage process (Lubliner et al. 1989). The common feature of the aforementioned methods is their use of the concept of a softening curve to arrive at the concrete fracture energies $G_{F}$ (the true fracture energy) and $G_{f}$ (the size effect fracture energy). The softening curve is defined by three material properties, the tensile strength $\left(f_{t}\right)$, the size effect fracture energy $\left(G_{f}\right)$ and the true fracture energy $\left(G_{F}\right)$, as shown in Fig. 1 .

The tensile strength $\left(f_{t}\right)$ can be obtained from a splitting tensile test (ASTM C496 2005; ACI 446 2009). The size effect fracture energy $\left(G_{f}\right)$ and the true fracture energy $\left(G_{F}\right)$ can be found from a three point bending test of a notched concrete beam (Maturana et al. 1990; Guinea et al. 1994; Planas et al. 1999; Kitsutaka et al. 1998; Elices et al. 2002; RILEM 2007; ACI 446 2009). The direct method of determining the tensile softening curve of concrete is a stable

\footnotetext{
${ }^{1)}$ Department of Civil Engineering, Korea Maritime and Ocean University, Busan 606-794, Republic of Korea. *Corresponding Author; E-mail: leonlee397@gmail.com ${ }^{2)}$ Department of Civil \& Environmental Engineering, The Pennsylvania State University, University Park, PA 16801, USA.

Copyright $($ The Author(s) 2014. This article is published with open access at Springerlink.com
}

tensile test, however this procedure has drawbacks such as the crack location not being known (Reinhardt et al. 1986; Bažant and Planas 1998). Accordingly, many researchers prefer indirect procedures such as the three-point bending test. The three-point bending test for the tensile properties of concrete is based upon the cohesive models of Hillerborg et al. (1976) which were further developed by Planas et al. (1999) and Guinea et al. (1994). Coronado and Lopez (2005, 2008) proposed experimental procedures for predicting the fracture behavior of externally bonded FRP strengthened concrete structures using three point bending tests and splitting tensile tests according to Guinea et al. (1994), Planas et al. (1999) and Elices et al. (2002). It was found that the bilinear softening curves approximated from the two tests successfully predicted the experimentally observed strain, strain distribution, failure loads and failure mode. Recently the ACI 446 committees collated research results and produced a draft ASTM test standard (ACI 446 2009) for the fracture toughness i.e. the fracture energy of concrete. The draft standard mentions that the precision and bias of the test methods are not yet known and that a consensus should be developed in order to publish an official ASTM standard for determining the tensile toughness of concrete (Gerstle 2010). Bažant and Yu (2011) recently pointed a nonuniqueness of cohesive softening law especially for small size concrete specimen $(D=25 \mathrm{~mm})$. For larger specimens ( $D=215 \mathrm{~mm})$, differences of load-CMOD graphs were negligible no matter what cohesive softening curves are used. Therefore, fracture test of specimens of one size is insufficient especially for small sized concrete structures. 


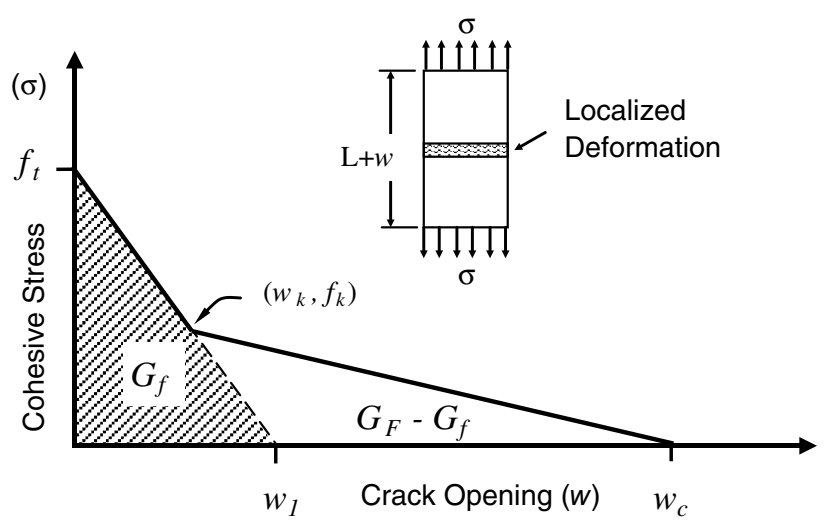

Fig. 1 Bilinear approximation of the softening curve (Coronado and Lopez 2008).

However, size effect testing could be avoided if following values are known a priori. It was recommended that $G_{F} / G_{f}$ and $\sigma_{1}$ (stress at kink point in the bilinear cohesive softening curve) $/ f_{t}$ are 2.5 and 0.25 , respectively.

\section{Objectives}

From the discussion above, it can be concluded that the standard method which is being developed for determining the fracture energy of concrete is not yet verified. Therefore, finding new test methods in order to develop a consensus on the standard test method are needed. This study aims to fulfill these needs. In this study, based on the RILEM (2007) and ACI 446 (2009) test methods, notched beams were tested using a three point bending frame. The sensitivity to the concrete fracture energy and the feasibility of the recommended end points (CMOD at $2 \mathrm{~mm}$ ) were investigated using data obtained from both the LVDT and crack mouth opening displacement (CMOD). A bilinear stress-crack opening curve is proposed based on the test results obtained. Some important findings from the tests performed are summarized in the conclusion section.

\section{Experiment Programs}

A total of 12 notched beams and 13 concrete cylinders were tested at two different times. These specimens (12 notched beams and 13 concrete cylinders) came from different concrete mixture trucks, using the same concrete mixture design, being poured the same day and at the same construction site. It should be noted that the concrete properties will be different depending on the variations of each of the concrete trucks. The quantity of entrained air was $4.5 \%$ of the volume of the concrete. A water to cement ratio of 0.5 was used. The maximum aggregate size was required to be equal or less than $25 \mathrm{~mm}$. According to RILEM (1990) recommendations, aggregate sizes in the range $1 \mathrm{~mm}$ to $25 \mathrm{~mm}$ are allowable for the beam size selected in this study. Likewise, RILEM report (2007) recommend of using a ligament of 4 times the aggregate size. At a NSF sponsored workshop in 2001 (Florida, USA), key researchers in the field of fracture toughness testing of concrete gathered and decided testing standards. A $150 \times 150 \times 525 \mathrm{~mm}$ notched beam for a three point bending test and a $150 \times 300 \mathrm{~mm}$ piece for a Brazilian splitting cylinder test were defined as the standard cases (ACI and ASTM, Gerstle 2010). The maximum allowable aggregate size for these tests was $25 \mathrm{~mm}$.

\section{Apparatus for the Bending Test}

The dimensions of the notched beam were a cross section of $152 \mathrm{~mm}$ by $152 \mathrm{~mm}$, a length of $560 \mathrm{~mm}$ and a notch depth of $33 \%$ of the beam depth. Using a brick sawing machine, $50 \mathrm{~mm}$ notches were made at mid span on the bottom side of the beams. An illustration of the beam is shown in Fig. 2. The apparatus for the three point bending tests on the notched beams was an MTS hydraulic test machine, which is operated by a closed loop control system. The $1,000 \mathrm{kN}$ load cell was replaced with a $100 \mathrm{kN}$ load cell (MTS-661-20E-03) to reduce measuring errors and to increase the accuracy since the maximum expected load from the three point beam tests would be less than $15 \mathrm{kN}$. The test frames were designed to withstand tests under $1,000 \mathrm{kN}$, however the load cell, which has a capacity of less than $100 \mathrm{kN}$, was used to accurately measure the applied load. Therefore, we see that the test setup is sufficiently rigid for one to perform three point bending tests on notched beams in order to obtain complete softening curves and that it provides stable test performances. The load cell (MTS 661-20E-03) has a resolution of $20 \mathrm{~N}$ and a nonlinearity of $0.08 \%$ of the $100 \mathrm{kN}$ full scale span. The clip on gage (Epsilon 3541-005M-100M-ST) has a resolution of $1.5 \mu \mathrm{m}$ and a non-linearity of $0.064 \%$ of the $10 \mathrm{~mm}$ full scale span. A 1 GPM servo valve was used for the MTS hydraulic test machine in order to provide a stable closed loop control. Two high precision LVDT with a range of $\pm 5 \mathrm{~mm}$ and a non-linearity of less than $0.2 \%$ of the full scale span were used for measuring the displacement. A total of 7 notched beams and 6 cylinders were tested under the stroke control test setup. Likewise, a total of 5 notched beams and 7 cylinders were tested under CMOD control.

\section{Splitting Tensile Test Setup}

To obtain softening curves for the concrete's tensional behavior, the maximum tensile strength of the concrete should be obtained at the same time and from the same batch. Instead of performing a direct tension test on plain concrete which is of needless difficulty, splitting tensile tests (ASTM C496) were conducted using $15 \mathrm{~cm}$ by $30 \mathrm{~cm}$ cylindrical concrete specimens. Some researchers have found that the test results could vary depending on the strip width/diameter of the cylinder $(b / D)$, the loading rate, geometry and the size effect (Rocco et al. 2001; Coronado and Lopez 2008) and it is therefore necessary to carefully consider these factors. Rocco et al. (2001) concluded that the load bearing strip as recommended by the ASTM standard 


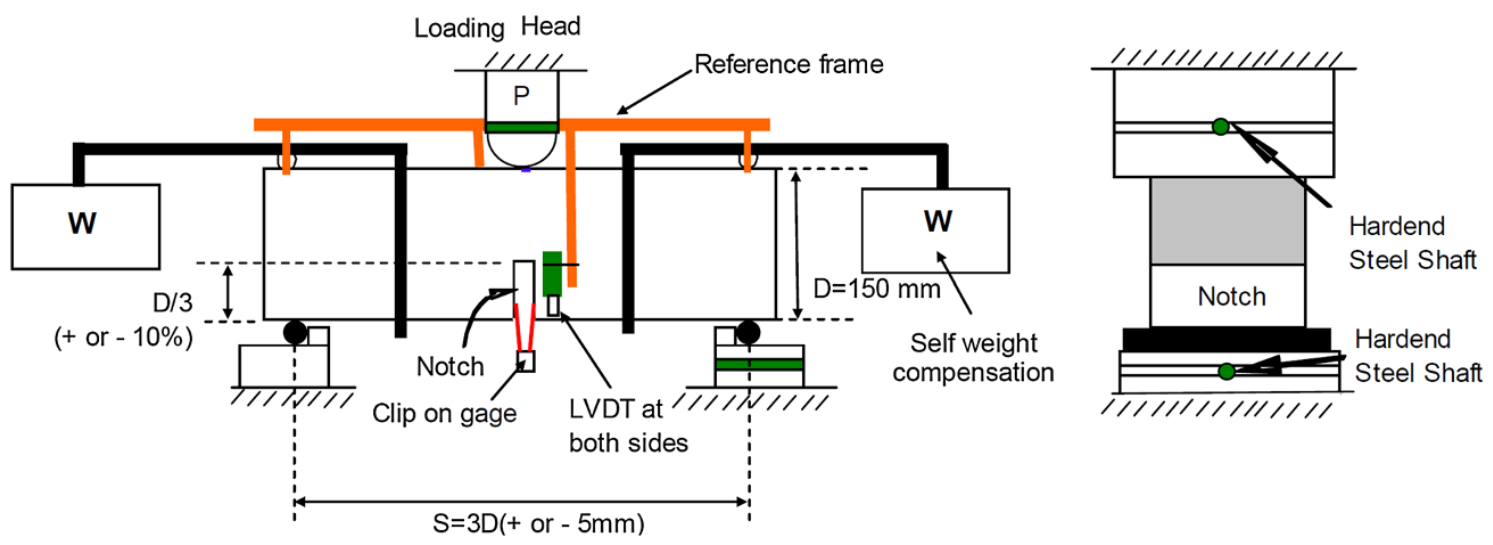

Fig. 2 Dimension of notched beam.

seem to be too wide, however it was found that if the width of the load bearing strip approaches zero then size effects vanish. Accordingly, Rocco et al. (2001) reported that those variations due to the strip width and the size effect could be minimized when the strip width is less than $4 \%$ of either the diameter or the width of the concrete specimens. A similar study was also performed by Coronado and Lopez (2008), who found that the primary crack would be noticeable only with a strip width in the range of $4-8 \%$ of the diameter of the cylinder. The primary crack was not arrested during the tests, indicating that the recorded maximum load could be regarded as a true maximum load. To minimize size effects, a diameter of $5 \%$ of the concrete cylinder was selected as the strip width for both the top and bottom loading points. Based on recommendations from ASTM C496, a loading rate of $50-100 \mathrm{kN} / \mathrm{min}$ was used during the tests.

\section{Three Point Bending Test Setup}

The dimensions for the notched beam and test procedures for the three point bending tests were designed based on recommendations from RILEM (2007) and ACI (2009). Two supports and a loading head were devised by the authors and fabricated by a local commercial fabricator (Sky top, PA, USA). Figure 3 shows the test setup and frames. One of the supports was designed to be free to rotate about any axis to minimize the torsional effects on tested beams. Ground hardened steel plates were also placed on the roller of the supports to prevent the concrete from crushing at the supports. A cylindrical loading head, which was also designed to be free to rotate to accommodate fabrication imperfections and to reduce the torsional effects on the loading plane was used. For stable test performance and in order to obtain reliable test data, the use of self-weight compensation is recommended (Bažant and Planas 1998). This can be done in various ways by using springs, double length specimens or a lever with a dead weight. In this study, a lever with dead weights was used, with the beam self weight being over compensated (see Fig. 3). Concrete micro cracks below the supports and the potential for a small gap between the steel plates in the loading frame can be a source of overestimation for the beam deflection, which can cause the overestimation of the concrete fracture energy. Therefore, as shown in Fig. 3, the reference frame was placed on top of the concrete beam and two LVDT were attached to the reference frame instead of the support. The reference frame was designed such that the LVDT were adjustable at any angle. Additionally, two aluminum plates were attached to the soffit of the concrete beam, providing a flat contact surface between the LVDT and were also used to secure a clip on gage.

For the stroke control loading procedure, it was programmed that a load of $5 \mathrm{kN}$ was incrementally applied onto the notched beam using a rate of $2.5 \mathrm{kN} / \mathrm{min}$ to remove geometrical mismatches and gaps. Secondly, a constant stroke control with a rate of $0.018 \mathrm{~mm} / \mathrm{min}$ was used until the end of the test. For the CMOD control loading procedure, a loading rate of $2 \mathrm{kN} / \mathrm{min}$ was used to remove geometrical mismatches and gaps until the load reached $2 \mathrm{kN}$. Then a constant CMOD loading rate $(0.01 \mathrm{~mm} / \mathrm{min})$ was used until the end of the test. Test results based on both stroke control and CMOD control will be compared and discussed in detail in a later section.

\section{Results of the Three Point Bending Tests}

Splitting tensile strengths were measured immediately after the three point bending tests. The tensile strength of the specimens was calculated using Rocco's empirical equation (Eq. 1), where $B$ and $D$ are the specimen dimensions, $P$ is the load and $\beta=b / D(5 \%)$ which is the relative width of the bearing strips. The overall average value of the tensile strength for all of the tested cylinders was $2.29 \mathrm{MPa}$.

$$
\sigma_{\max }=\frac{2 P}{\pi B D}\left(1-\beta^{2}\right)^{2 / 3} .
$$

Figure 4 shows a typical load-CMOD graph for the three point bending test. Micro-cracking started before the peak load, however the crack was not visible after the onset of cracking, and only appeared after around $0.5 \mathrm{~mm}$ of CMOD. Therefore, estimation of the crack propagation length could not be done by visual inspection. The overall load-CMOD curves shows smooth softening curves after the peak loads, indicating that tests were conducted under a stable test regime. The test was run until $2 \mathrm{~mm}$ of CMOD. The areas 


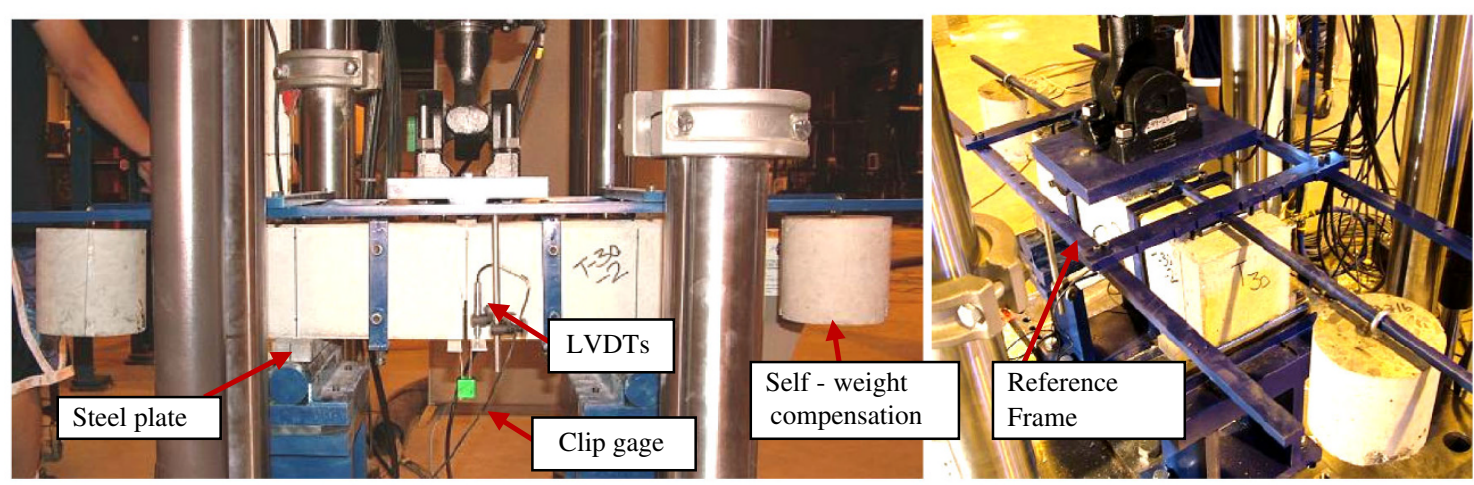

Fig. 3 Three-point bending test of a notched concrete beam: test set-up.

under the curves were calculated in order to find the fracture energy of the tested concrete.

Figure 5a explains the procedure designed for the stroke control. For the loading procedure of the stroke control, a $5 \mathrm{kN}$ load was gradually applied onto the notched beam using a rate of $2.5 \mathrm{kN} / \mathrm{min}$ in order to remove geometrical mismatches and gaps. The stroke control became linear after an initial load of $5 \mathrm{kN}$ was applied, indicating that a constant rate was used for the stroke. However, it is interesting to note that although the stroke rate was constant $(0.018 \mathrm{~mm} / \mathrm{min})$, the corresponding LVDT and CMOD rates were changing during the tests, especially after the onset of cracking. At the onset of cracking, the CMOD and LVDT rates changed drastically, indicating that the crack opening rate could be changed despite a constant loading rate from the stroke being used. This could be an indication that constant CMOD and constant stroke may cause differing test results.

Figure $5 \mathrm{~b}$ explains the CMOD control test. A loading rate of $2 \mathrm{kN} / \mathrm{min}$ was used to remove any geometrical mismatches and gaps until the load reached $2 \mathrm{kN}$, and then a constant CMOD loading rate $(0.01 \mathrm{~mm} / \mathrm{min})$ was maintained until the end of the test. It can be seen that the rates of CMOD are constant until the end of the test, however the slopes for the LVDT and the stroke changed at the onset of the cracking. It should be noted that the test will never reach the loading level of zero as complete failure of the beam is approached asymptotically (Petersson 1981). This implies that the fracture test for a concrete beam is stopped before total energy dissipation. The draft ASTM standard recommends the use of $2 \mathrm{~mm}$ as an end point for the test (ACI 446 2009) and the fracture energy is calculated at this point.

Figure 6 shows load-CMOD graphs for the tested notched beams under the stroke control procedure. Results from all of the tested beams are presented in Table 1. The following nomenclature is used in this study to refer to each specimen: TO-O, where the first digit indicates the truck number, followed by a number for each specimen tested. For each of the test series, the general trend among specimens is very similar. This is an indication of the test repeatability, and the correctness of the procedures. The observed peak loads exhibit variation and it is thought that the aggregate alignment and density of the aggregate around the notched area can affect the peak load. Likewise, a total of 5 notched

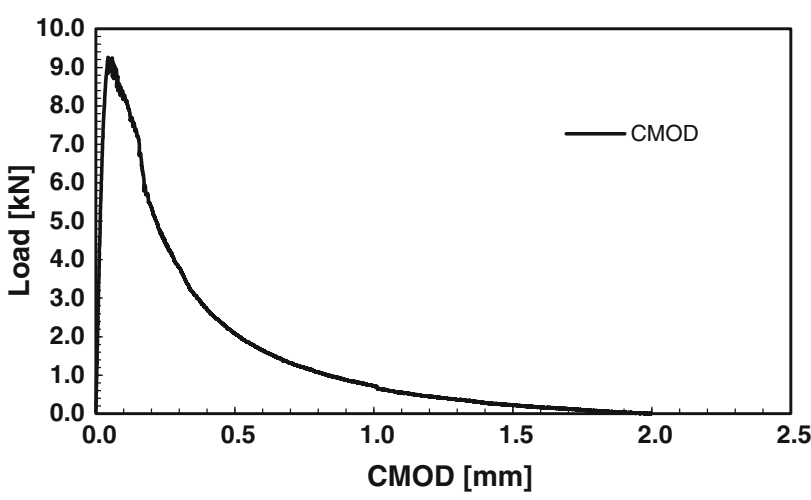

Fig. 4 A typical load versus CMOD graph under stroke control.

beams were tested under the CMOD control procedures. The test was performed up to $5 \mathrm{~mm}$ of CMOD, however the end point for the test was selected as being $2 \mathrm{~mm}$ of CMOD in order to be compared with specimens tested under stroke control (see Fig. 7). Except for specimen T30-3, which was expected to have a lower fracture energy, similar softening curves were observed from all of the specimens.

\section{Obtained Fracture Energy of Concrete}

The experimental results were used to find both the fracture energy of the concrete and the elastic modulus (Elices et al. 1992; Bažant and Planas 1998; RILEM 1990; RILEM report 2007). The elastic modulus could be obtained from the initial compliance calculated from the positively sloped section of the load-CMOD curve. The load-displacement curve is used for calculation of the works of fracture, a measured work of fracture can be obtained from the area under the curve. Since complete failure of the beam in the three point bending test is approached asymptotically (Petersson 1981), an end point for the test could not be determined. Therefore, the test needed to be stopped at some point before complete fractures, meaning that the measured work of fracture does not represent the true fracture energy of the tested concrete. In order to overcome this Elices et al. (1992) proposed a method for estimating the true fracture energy from the tested fracture energy using a far tail 


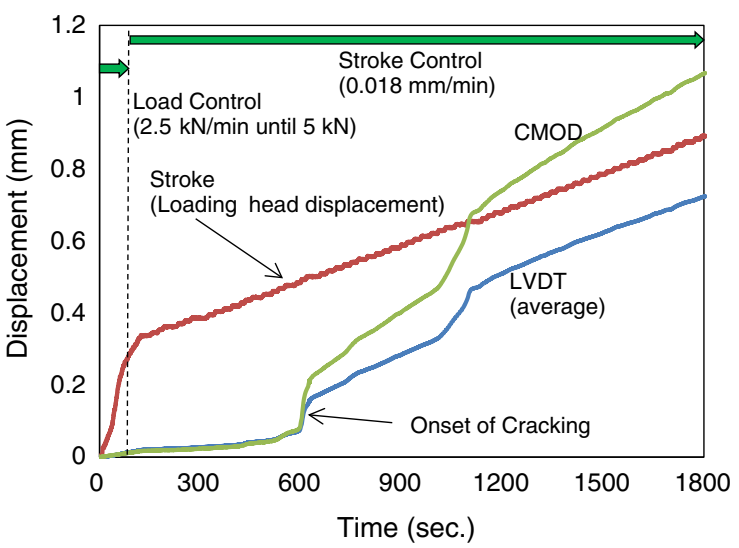

(a)

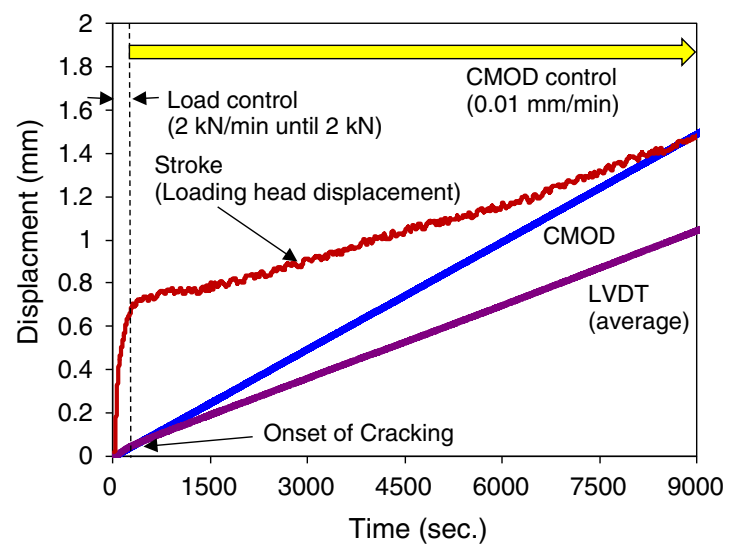

(b)

Fig. 5 Comparison of the loading rate between stroke (a) and CMOD control (b).

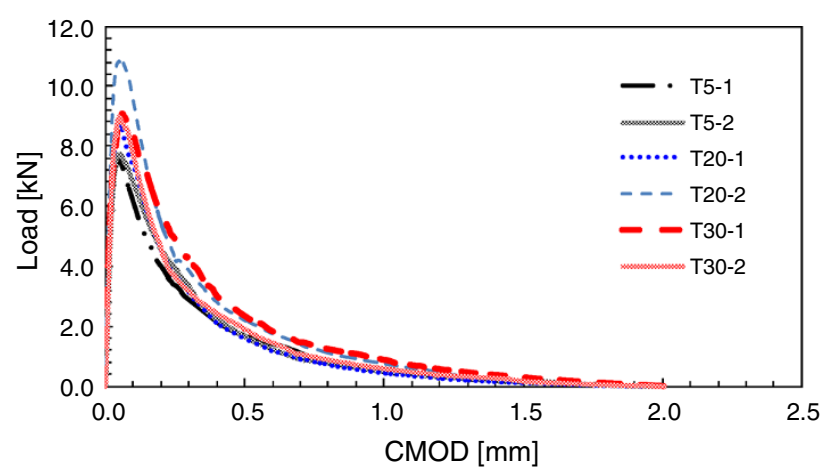

Fig. 6 The recorded CMOD versus load graph under stroke control.

constant value $(A)$. The far tail constant value $(A)$ can be obtained approximately from least squares fitting of the curve obtained from the three point bending test. Figure 8 shows one example of how to obtain the far tail constant value $(A)$. Here $u_{0}$ indicates the initial LVDT or CMOD data and $u_{r}$ indicates the LVDT or CMOD data at the residual load for the rising part of the curve. The slope of the graph obtained was the value $A\left(1,074 \mathrm{Nm}^{2}\right)$ of the tested specimen. Since the test data was obtained based upon a $100 \mathrm{~Hz}$ acquisition rate, in order to capture any sudden changes during a test, 50 points of the test data were averaged. The obtained fracture energy and the related parameters are shown in Table 1. The elastic modulus $(E)$, fracture energy, area below the softening curve $\left(G_{F M}\right)$, the size effect fracture energy $\left(G_{f}\right)$ and the true fracture energy $\left(G_{F}\right)$ are listed. $G_{F M}$ is not a true fracture energy. By using constant value $(A)$, corrected true fracture energy $\left(G_{F}\right)$ can be estimated.

\section{Sensitivity Study on the Far Tail Constant $(A)$}

Originally, Petersson (1981) and Elices et al. (1992) used LVDT data to calculate the far tail constant $(A)$, however the ACI 446 (2009) test method uses CMOD data to calculate the far tail constant $(A)$. These studies led the authors to check the differences of the fracture energies $\left(G_{F}\right.$ and $\left.G_{f}\right)$, when the far tail constant $A$ is calculated by LVDT or CMOD.

Table 1 and Fig. 9 show the differences of the fracture energies $G_{F}$ and $G_{f}$ from both the LVDT and CMOD test data. From this set of experimental results, it can be seen that the calculated true fracture energy $\left(G_{F}\right)$ is very sensitive to the far tail constant value $(A)$. A far tail constant $(A)$ was calculated using the recorded CMOD data tends to yield a larger fracture energy $\left(G_{F}\right)$ than that calculated using recorded LVDT data. It is interesting to note however that the size effect fracture energy $\left(G_{f}\right)$ was not changed.

\section{Sensitivity Study on Different Loading Conditions}

The true fracture energy $\left(G_{F}\right)$ is calculated using the measured fracture energy $\left(G_{M}\right)$ plus the estimated tail fracture energy $\left(G_{\text {tail }}\right)$ using the far tail constant value. The fraction of the tail fracture energy $\left(G_{\text {tail }}\right)$ calculated using the $A$ value under stroke control and CMOD control was calculated. Elices et al. (1992) concluded that the correction of $G_{F}$ calculated using the constant $A$ value could be as large as $20 \%$ of the total fracture energy of the concrete. In this study, the tail fracture energy $\left(G_{\text {tail }}\right)$ calculated from the far tail constant $(A)$ was in the range between 18 and $35 \%$, which is a larger percentage than the value that Elices et al. (1992) used in their study (see Fig. 10). The large aggregate size of the tested specimen is considered to have caused this larger fraction of the tail fracture energy. The far tail constant $(A)$ also affects the fraction of the tail fracture energy $\left(G_{\text {tail }}\right)$. As observed from the previous comparison of $G_{F}$, a value of $A$ calculated from CMOD data also tends to have a larger tail fracture energy $\left(G_{\text {tail }}\right)$.

\section{Sensitivity Study on the End Points for the Three Point Bending Tests}

The load-CMOD curve for the notched beam behaves asymptotically, as noted by Petersson (1981) and later 
Table 1 Analysis of obtained test results with far tail constant A.

\begin{tabular}{|c|c|c|c|c|c|c|c|c|}
\hline \multicolumn{3}{|c|}{ LVDT and CMOD data } & \multicolumn{3}{|c|}{ LVDT data } & \multicolumn{3}{|c|}{ CMOD data } \\
\hline Specimen & $E(\mathrm{GPa})$ & $G_{F M}(\mathrm{~N} / \mathrm{m})$ & $A\left(\mathrm{~N} \mathrm{~mm}^{2}\right)$ & $G_{F}(\mathrm{~N} / \mathrm{m})$ & $G_{f}(\mathrm{~N} / \mathrm{m})$ & $A\left(\mathrm{~N} \mathrm{~mm}^{2}\right)$ & $G_{F}(\mathrm{~N} / \mathrm{m})$ & $G_{f}(\mathrm{~N} / \mathrm{m})$ \\
\hline \multicolumn{9}{|c|}{ Stroke } \\
\hline T5-1 & 38.87 & 122.29 & 353 & 154.84 & 25.83 & 730 & 173 & 26.05 \\
\hline T5-2 & 35.34 & 133.35 & 419 & 171.25 & 32.3 & 787 & 184.14 & 32.15 \\
\hline T20-1 & 37.41 & 127.92 & 315 & 157 & 71.23 & 626 & 168.35 & 71.08 \\
\hline $\mathrm{T} 20-2$ & 42.67 & 175.46 & 530 & 223.07 & 153.64 & 999 & 240.09 & 153.17 \\
\hline T30-1 & 34.55 & 185.99 & 839 & 261.82 & 73.7 & 1448 & 279.58 & 72.05 \\
\hline T30-2 & 35.7 & 132.29 & 384 & 169.03 & 75.58 & 811 & 184.68 & 75.36 \\
\hline Avg. & 37.42 & 146.22 & 473 & 189.50 & 72.05 & 900 & 204.97 & 71.64 \\
\hline \multicolumn{9}{|c|}{ CMOD } \\
\hline T5-3 & 30.6 & 128.17 & 289 & 156.33 & 57.22 & 648 & 169.99 & 57.14 \\
\hline T20-3 & 36.42 & 155.78 & 509 & 202.83 & 71.94 & 1074 & 221.61 & 71.71 \\
\hline T20-4 & 29.14 & 143.19 & 648 & 202.23 & 52.34 & 1200 & 220.65 & 51.65 \\
\hline T28-1 & 31.32 & 194.87 & 497 & 239.44 & 123.89 & 864 & 250.64 & 122.34 \\
\hline T30-3 & 38.11 & 103.8 & 198 & 123.17 & 61 & 446 & 132.56 & 60.92 \\
\hline Avg. & 33.12 & 145.16 & 428 & 184.80 & 73.28 & 836 & 199.09 & 72.75 \\
\hline
\end{tabular}

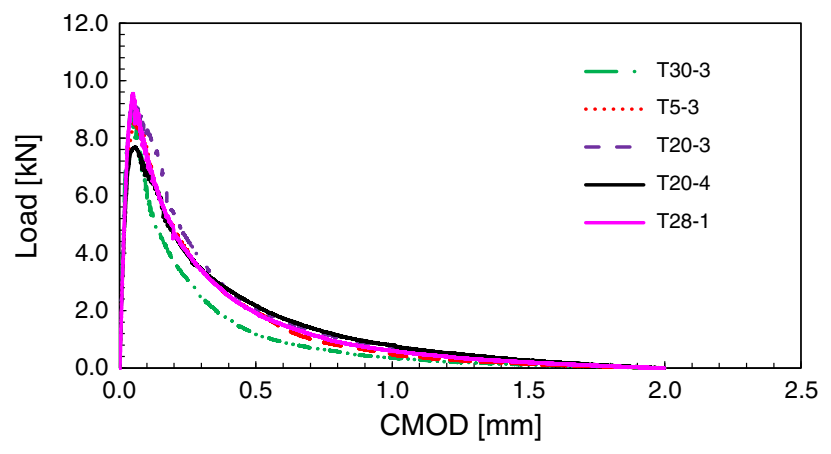

Fig. 7 Recorded CMOD versus load graph under CMOD control.

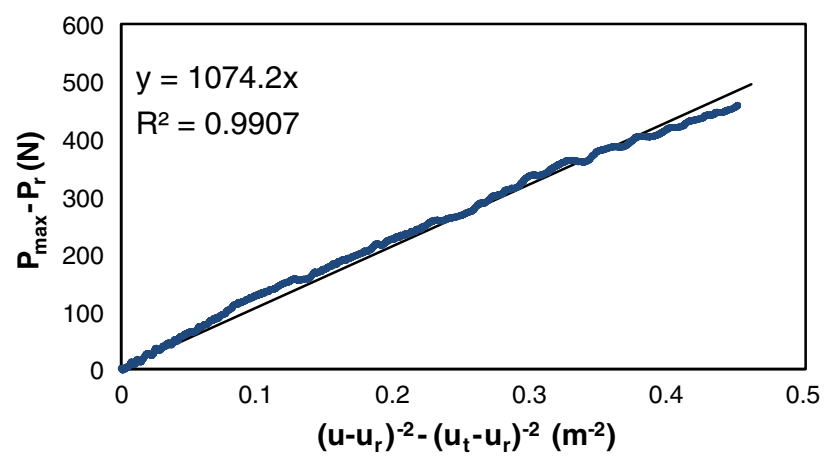

Fig. 8 Determination of a far tail constant $A$ value.

confirmed by Elices et al. (1992). Therefore, the applied load for the notched beam will never reach a zero value since the load is asymptotically approaching the zero value. This phenomenon indicates that the bending test for a notched concrete beam is stopped before complete failure and that therefore, the selection of the end point is important for estimating the fracture energy of the concrete. Conventionally, $2 \mathrm{~mm}$ of CMOD is considered to be the end of the test. If the measurements of the fracture energy for the three cases $(3,4$ and $5 \mathrm{~mm}$ ) show consistency with the $2 \mathrm{~mm}$ results, Petersson's asymptotic curve for predicting the tail fracture energy $\left(G_{\text {tail }}\right)$ would be proven to be the correct procedure. Figure 11 shows the load-CMOD curve obtained. This curve was modified to finish the tests at 2, 3, 4 and $5 \mathrm{~mm}$. A test beyond $5 \mathrm{~mm}$ could not be considered since all of the tests were stopped at $5 \mathrm{~mm}$ of CMOD in order to ensure stable test conditions. Figure 12 shows the results obtained for values of the true fracture energy $\left(G_{F}\right)$, the size effect fracture energy $\left(G_{f}\right)$ and the measured fracture energy $\left(G_{M}\right)$. In a previous section the true fracture energy $\left(G_{F}\right)$ and the size effect fracture energy $\left(G_{f}\right)$ were explained. The measured fracture energy $\left(G_{M}\right)$ indicates the fracture energy which is measured when based upon a selected end point and is therefore not the true fracture energy. The measured fracture energy $\left(G_{M}\right)$ would change if different end points were selected. The measured fracture energies $\left(G_{M}\right)$ that were calculated using the area under the curve are shown in Table 2 against the value of the end point. As Fig. 12 showed, larger values for the end points tend to yield larger values for the measured fracture energy $\left(G_{M}\right)$, and as a result the true fracture energy $\left(G_{F}\right)$ became larger due to the addition of a larger value for the measured fracture energy $\left(G_{M}\right)$. Larger end points, will without fail give larger measured fracture energy $\left(G_{M}\right)$ values since the area under the curve increases.

However, the final true fracture energy $\left(G_{F}\right)$ should be consistent, no matter what end point is chosen, provided that 

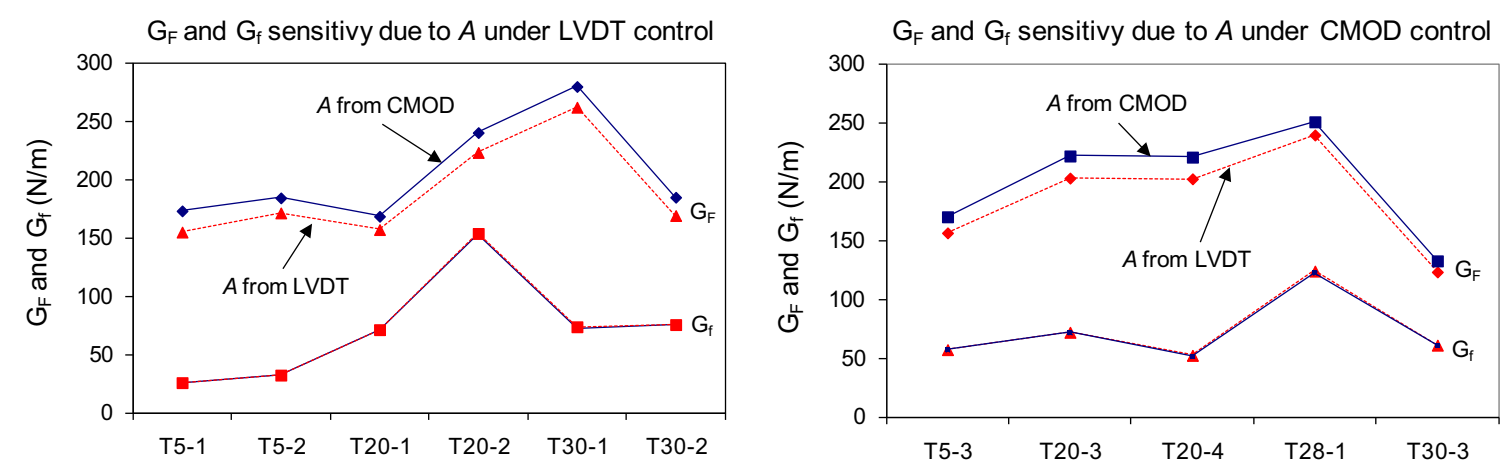

Fig. 9 Fracture energy sensitivity due to constant $A$.
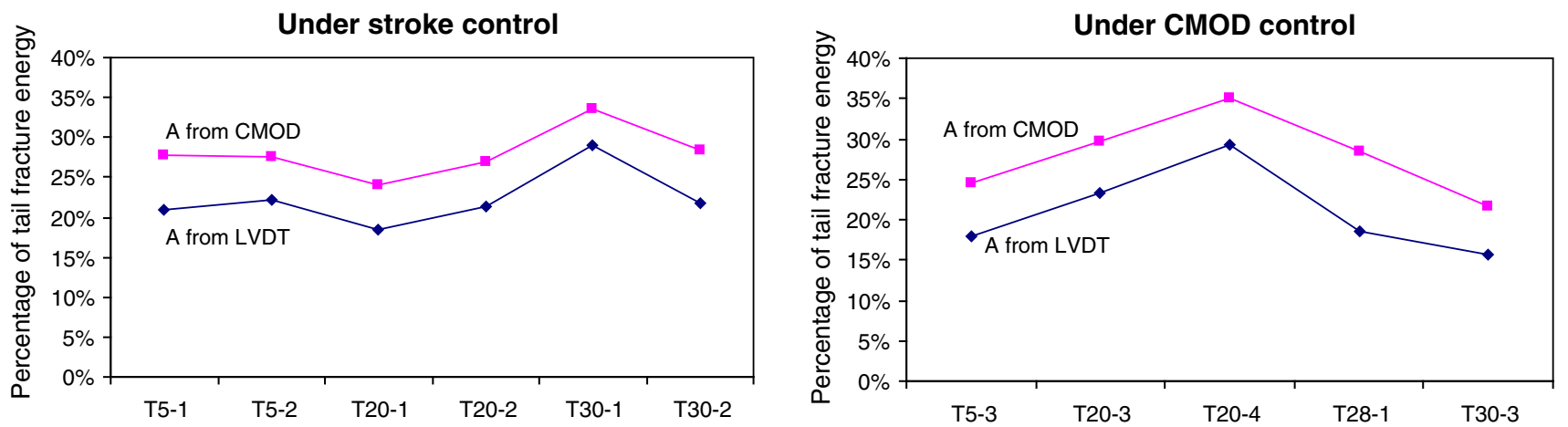

Fig. 10 Percentage of tail fracture energy.

the test follows the same asymptotic curves. If the final true fracture energies depend on the end points and show some variation, then Peterson's asymptotic assumption (Petersson $1981)$ to predict the tail fracture energy $\left(G_{t a i l}\right)$ is not fit for the specimens in this study, given their larger aggregate size $(25 \mathrm{~mm})$. The results obtained show that selections with a larger number of end points tend to yield larger true fracture energies $\left(G_{F}\right)$. It is also interesting to see that end points between 3 and $4 \mathrm{~mm}$ show relatively similar values to those at 1 and $5 \mathrm{~mm}$. This indicates that there should be an appropriate end point for each different aggregate size. Therefore, if the size of the aggregate increases, then the end point should be increased in order to get the precise fracture energy. However, it was observed that the size effect fracture energy $\left(G_{f}\right)$ was not sensitive to the selection of the end point since the tail part of the load-CMOD curves are more related to the true fracture energy $\left(G_{F}\right)$.

\section{Proposed Bilinear Stress-Crack Opening Displacement Curves}

The fracture energy of the concrete which was previously obtained is used to determine the bilinear stress-crack opening displacement curves (see Fig. 1) according to Guinea's bilinear approximation (1994). The bilinear softening curves (bilinear stress-crack opening displacement curves) have been used for modeling concrete fracture behaviors based on the cohesive crack model. A detailed procedure for approximating the bilinear softening curve from loadCMOD data can be found in many sources (RILEM 1990;

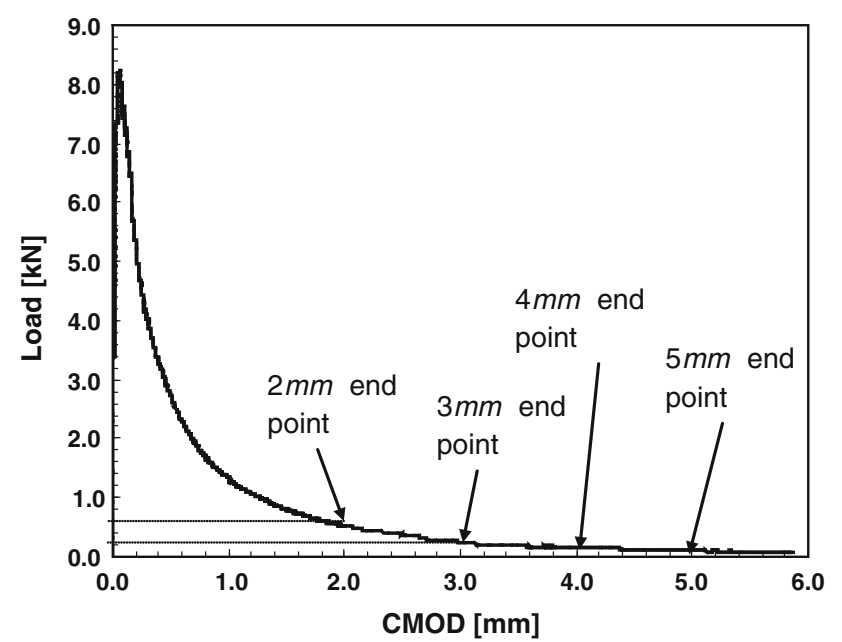

Fig. 11 Load-CMOD curves of T20-4 and designated ending point.

Guinea et al. 1994; Planas et al. 1999; Elices et al. 2002; RILEM report 2007; ACI 446 2009) and so the relevant aspects are only summarized here. Guinea et al. (1994) proposed the bilinear approximation based upon the cohesive crack model. The bilinear curve can be shaped based on four parameters: the tensile strength of concrete $\left(f_{t}\right)$, the true fracture energy $\left(G_{F}\right)$, the abscissa of the centroid of the softening curve $(\bar{w})$, and the initial tangent intercept $\left(w_{1}\right)$. The tensile strength of the concrete $\left(f_{t}\right)$ can be obtained from the splitting tensile test. The true fracture energy of the concrete is obtained from the area enclosed beneath the softening curve as measured by the RILEM method (2007). 


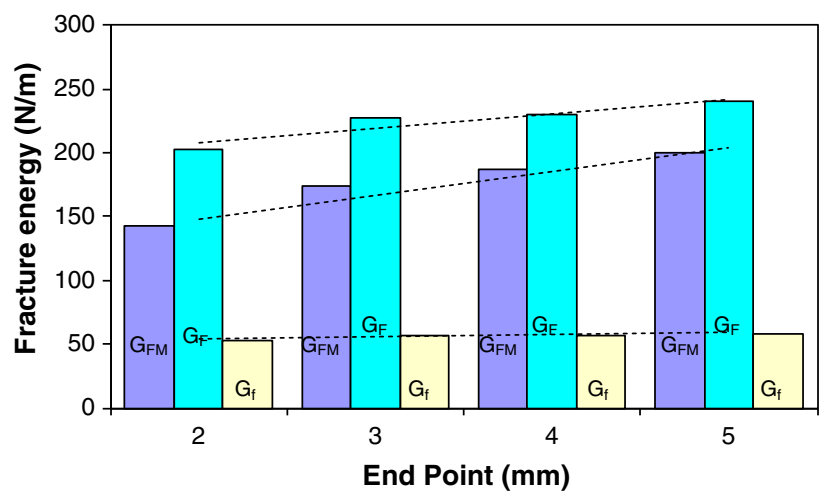

Fig. 12 Load-CMOD curves of T20-4 and designated ending point.

The abscissa of the centroid of the softening curve $(\bar{w})$ can be obtained from the tail part of the load-displacement. The tail part of the load-displacement curve can be modeled by rigid body kinematics as explained in Guinea et al. (1994). The initial tangent intercept $\left(w_{1}\right)$ can be calculated from the brittleness length, the mean elastic modulus and the tensile strength of the concrete according to Planas et al. (1999). The detailed theory based upon rigid body kinematics and the derived equations can be found in the aforementioned sources (Guinea et al. 1994; Planas et al. 1999; Bažant and Planas 1998; Elices et al. 2002; RILEM 2007) and the draft ASTM test standard for the fracture toughness testing of concrete (ACI 446 2009). The far tail constant $(A)$ and the elastic modulus $(E)$, obtained from the ascending branch of the load-CMOD data, were used. The critical crack opening $\left(w_{c}\right)$ and the kink point were determined and the maximum stress is simply obtained from the splitting tensile test. An overall bilinear graph was drawn instead of showing each bilinear curve from each truck (batch). In Fig. 13, the two stress-crack opening bilinear graphs from the specimens tested under stroke control and under CMOD control were compared to the bilinear stress-crack opening graphs determined by the CEB-FIP $(1990,2010)$ model code. Based on the CEB-FIP model code published in 1990, a coefficient of maximum aggregate size $(25 \mathrm{~mm})$ was considered to follow the specifications suggested by the CEB-FIP (1990) model code. The coefficient of maximum aggregate size (25 mm) was selected by interpolating between the $16 \mathrm{~mm}$ and the $32 \mathrm{~mm}$ aggregate size since there is no coefficient for an aggregate with a $25 \mathrm{~mm}$ maximum size. The stress at the kink point is estimated as being $15 \%$ of the maximum tensile stress. For the CEB-FIP model code published in 2010, a coefficient related to the maximum aggregate size is not suggested. Instead, one coefficient value of 5 , is proposed for all cases. The stress at the kink point is estimated at $20 \%$ of the maximum tensile stress. The final forms of the bilinear softening curves from both experiments and from the CEB-FIP model code are shown in Fig. 13. The final bilinear form for the CMOD control and stroke control show a similar shape, indicating that the loading rate would not be a significant factor in order to control the final form of the bilinear softening curves. Both of the CEB-FIP $(1990,2010)$ models produced larger size effect fracture energy $\left(G_{f}\right)$ and smaller tail fracture energy $\left(G_{\text {tail }}\right)$ than for those obtained using the bilinear curves (stroke and CMOD controls) from experiments.

Based on the experimental results obtained in this study, the CEB-FIP (2010) model code case is closer to the experimentally obtained bilinear curves for crack openings between 0 and $100 \mu \mathrm{m}$. On the other hand, the CEB-FIP (1990, 2010) model code is closer to the experimentally obtained bilinear curves for crack openings between 400 and $500 \mu \mathrm{m}$ as shown in Fig. 13. The critical crack opening displacement is estimated using the aggregate size in CEBFIP $(1990,2010)$ and so as an approximation a coefficient 6 is used. CEB-FIP (2010) recommends using a coefficient value of 5 for any maximum aggregate size. As a result of the simplification the critical crack opening displacement as estimated by CEB-FIP (1990, 2010) is closer to the experimentally obtained bilinear curve. This leads the authors to propose the following bilinear stress crack opening displacement curve based on CEB-FIP $(1990,2010)$ and the obtained test results (see Fig. 14). The aggregate size should be considered according to CEB-FIP (1990, 2010), with the stress at the kink point being determined as being $20 \%$ of the maximum tensile strength according to CEB-FIP (2010). The crack opening displacement at the kink point is adjusted to fit the experimental results obtained in this study. However, the development of a standard test method for the concrete fracture toughness is still in progress, therefore, in this study the latest consensus on the test method was used and the proposed bilinear curves were obtained. Since the biases of the test are still unknown, sufficient research results and verifications on the proposed bilinear curves should be obtained in order to gain a consensus on the best test method for concrete toughness. As a future study, a large number of fracture tests using the test setup proposed in this study should be performed in order to verify the test method for

Table 2 Analysis of obtained test results with far tail constant A of CMOD.

\begin{tabular}{c|c|c|c|c|c}
\hline Specimen & End point $(\mathrm{mm})$ & $G_{F M}(\mathrm{~N} / \mathrm{m})$ & $A\left(\mathrm{~N} \mathrm{~mm}^{2}\right)$ & $G_{F}(\mathrm{~N} / \mathrm{m})$ & $G_{f}(\mathrm{~N} / \mathrm{m})$ \\
\hline \hline T20-4 & 2 & 143.19 & 648 & 202.23 & 52.34 \\
\hline $\mathrm{T} 20-4$ & 3 & 173.52 & 903 & 227.74 & 56.84 \\
\hline $\mathrm{T} 20-4$ & 4 & 187.38 & 951 & 230.05 & 57.18 \\
\hline $\mathrm{T} 20-4$ & 5 & 200.49 & 1092 & 239.7 & 58.28 \\
\hline
\end{tabular}



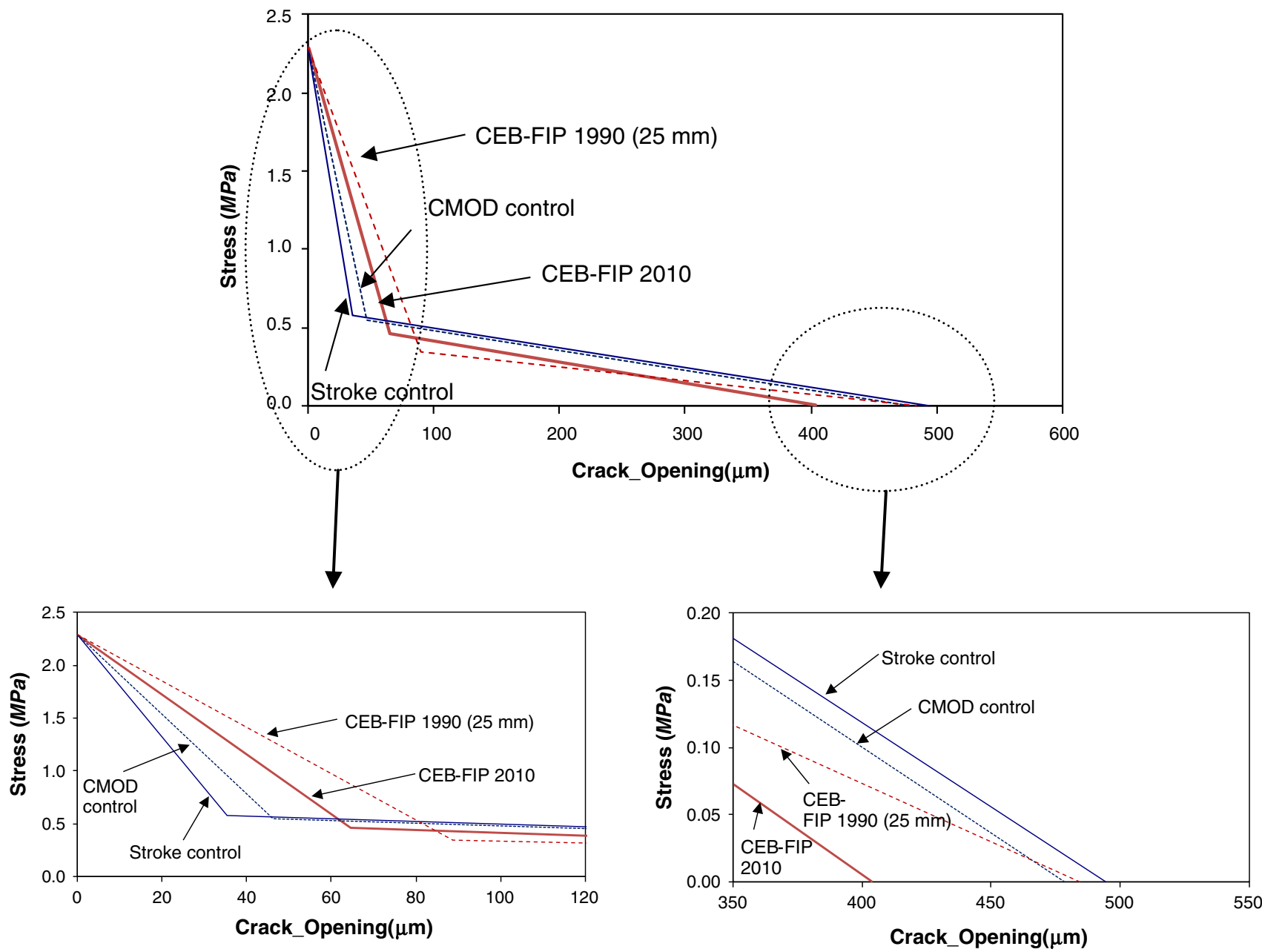

Fig. 13 Bilinear curve comparison.
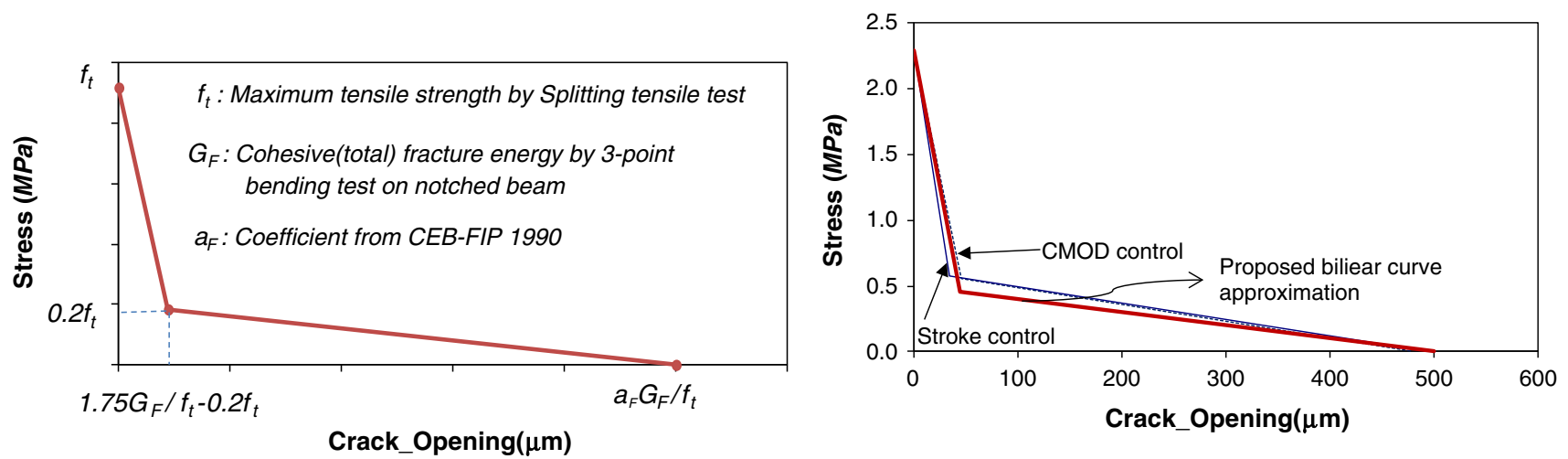

Fig. 14 Proposed bilinear curve and a comparison with other bilinear curves.

concrete fracture toughness and the proposed bilinear softening curves in this study.

\section{Conclusions}

According to the concrete toughness (fracture energy) tests performed in this study, conclusions are derived as follows:

- The average true fracture energies are 189 and $185 \mathrm{~N} / \mathrm{m}$ from the stroke control and the CMOD control, respectively, after considering the possible sources of energy dissipation. The final forms of the bilinear stresscrack opening curves of concrete from both loading rates were also very similar.

- It was found that the fracture energy could be sensitive to the far tail constant $(A)$ value and to the selection of the end points used to calculate the true fracture energy. When CMOD data was used to calculate $A$, a maximum increase of $11 \%$ of the true fracture energy $\left(G_{F}\right)$ was found. However, the size effect fracture energy $\left(G_{f}\right)$ did not change with the value of $A$. 
- It was also observed that an end point of $5 \mathrm{~mm}$ yields an $18 \%$ larger true fracture energy $\left(G_{F}\right)$ than that of the $2 \mathrm{~mm}$ end point. Therefore, appropriate selection of the end point of the test should be considered along with the maximum aggregate size in order to obtain the true fracture energies. However, the size effect fracture energy $\left(G_{f}\right)$ was not influenced by the selection of the end point.

- It is concluded that the loading rate is not a significant factor to control the fracture energy of the concrete as well as the final forms of the bilinear softening curves.

- The overall shape of the bilinear stress crack opening curve estimated by the CEB-FIP model is similar to the experimentally obtained bilinear curves. However, it tends to exhibit smaller tail fracture energy $\left(G_{\text {tail }}\right)$ and a larger size effect fracture energy $\left(G_{f}\right)$ when compared to the experimental ones.

- In this study, a new bilinear curve is proposed based upon both the CEB-FIP recommended bilinear curves and experimentally obtained bilinear curves. The proposed bilinear curve in this study fits the experimental bilinear curves well.

- The accuracy of the size effect fracture energy $\left(G_{f}\right)$ determined using one size of notched beam and one size of cylinder has recently been brought into question. As a further study, a comparison of the size effect fracture energy $\left(G_{f}\right)$ as determined using multiple sizes of notched beams along with the results obtained from this study is recommended.

- The biases of the various concrete toughness tests developed is still unknown. Sufficient data should be gathered and sufficient research conclusions should be collected in order to define a reliable test standard. It is hoped that results obtained from this study might be helpful for building a consensus on the concrete fracture toughness test method.

\section{Acknowledgment}

This study is based upon work supported by the National Science Foundation under a CAREER Grant No. 0330592.

\section{Open Access}

This article is distributed under the terms of the Creative Commons Attribution License which permits any use, distribution, and reproduction in any medium, provided the original author(s) and the source are credited.

\section{References}

ACI 446. (2009). Fracture toughness testing of concrete. Farmington Hills, MI: America Concrete Institute (in progress).
ASTM. (2005). Standard test method for splitting tensile strength of cylindrical concrete specimens. Annual book of ASTM standards, C496/C496M (Vol. 04.02).

Bažant, Z. P. (1976). Instability, ductility, and size effect in strain softening concrete. Journal of Engineering Mechanics Division, 102(2), 331-344.

Bažant, Z. P., \& Planas, J. (1998). Fracture and size effect in concrete and other quasibrittle materials. Boca Raton, FL: CRC Press.

Bažant, Z. P., \& Yu, Q. (2011). Size effect testing of cohesive fracture parameters and nonuniqueness of work-of-fracture method. Journal of Engineering Mechanics, 137(8), $580-588$.

CEB-FIP. (1990). Final draft CEB-FIP model code 1990. Bulletin Information Committee Euro-International, Beton 203

CEB-FIP. (2010). Final draft CEB-FIP model code 2010. Bulletin Information Committee Euro-International. Beton 203.

Coronado, C., \& Lopez, M. (2005). Modeling of FRP-concrete bond using nonlinear damage mechanics. Proceedings of the FRPRCS-7: 7th International symposium on fiber reinforced polymer reinforcement for reinforced concrete structures, ACI, KS.

Coronado, C. A., \& Lopez, M. M. (2008). Experimental characterization of concrete epoxy interfaces. Journal of Materials in Civil Engineering, 20(4), 303-312.

Elices, M., Guinea, G., \& Planas, J. (1992). Measurement of the fracture energy using 3-point bend tests. 1. Influence of experimental procedures. Materials and Structures, 25(148), 212-218.

Elices, M., Guinea, G. V., Gomez, J., \& Planas, J. (2002). The cohesive zone model: Advantages, limitations and challenges. Engineering Fracture Mechanics, 69(2), 137-163.

Gerstle, W. (2010). Progress in developing a standard fracture toughness test for concrete. Structures Congress 2010, ASCE, Orlando, FL.

Guinea, G., Planas, J., \& Elices, M. (1994). A general bilinear fitting for the softening curve of concrete. Materials and Structures, 27(2), 99-105.

Hillerborg, A., Modeer, M., \& Petersson, P. (1976). Analysis of crack formation and crack growth in concrete by means of fracture mechanics and finite elements. Cement and Concrete Research, 6(6), 773-782.

Kitsutaka, Y., Kurihara, N., \& Nakamura, S. (1998). Evaluation method of tension softening properties. Proceedings of the FRAMCOS 3 preconference workshop on quantitative evaluation methods for toughness and softening properties of concrete, Gifu, Japan.

Lubliner, J., Oliver, J., Oller, S., \& Onate, E. (1989). Plasticdamage model for concrete. International Journal of Solids and Structures, 25(3), 299-326.

Maturana, P., Planas, J., \& Elices, M. (1990). Evolution of fracture behaviour of saturated concrete in the low temperature range. Engineering Fracture Mechanics, 35(4-5), 827-834

Petersson, P. E. (1981). Crack growth and development of fracture zones in plain concrete and similar materials. Rep. 
TVBM-1006, Division of Building Materials, Lund Institute of Technology, Sweden.

Planas, J., Guinea, G. V., \& Elices, M. (1999). Size effect and inverse analysis in concrete fracture. International Journal of Fracture, 95(1-4), 367-378.

Planas, J., Guinea, G. V., Galvez, J. C., Sanz, B., \& Fathy, A. M. (2007). Indirect test for stress-crack opening curve. RILEM report-TC-187-SOC.

Reinhardt, H. W., Cornelissen, H. A. W., \& Hordijk, D. A. (1986). Tensile tests and failure analysis of concrete. ASCE Journal of Structural Engineering, 112(11), 2462-2477.
RILEM Draft Recommendation. (1990). Determination of fracture parameter $\left(\mathrm{K}_{\mathrm{ic}}^{\mathrm{s}}\right.$ and $\left.\mathrm{CTOD}_{\mathrm{c}}\right)$ of plain concrete using three point bend tests. Materials and Structures, 23, 457-460.

Rocco, C., Guinea, G. V., Planas, J., \& Elices, M. (2001). Review of the splitting-test standards from a fracture mechanics point of view. Cement and Concrete Research, 31(1), 73-82. 